\title{
Editorial
}

\section{Recent Advances in Scheduling and Its Applications}

\author{
Peng Liu, ${ }^{1}$ Hua Gong, ${ }^{2}$ Baoqiang Fan, ${ }^{3}$ Radoslaw Rudek, \\ Purushothaman Damodaran, ${ }^{5}$ and Guochun Tang ${ }^{6}$ \\ ${ }^{1}$ School of Management, Shenyang University of Technology, Shenyang, Liaoning 110870, China \\ ${ }^{2}$ School of Science, Shenyang Ligong University, Shenyang, Liaoning 110159, China \\ ${ }^{3}$ School of Mathematics and Statistics, Ludong University, Yantai, Shandong 264025, China \\ ${ }^{4}$ Institute of Business Informatics, Wrocław University of Economics, Komandorska 118/120, 53-345 Wrocław, Poland \\ ${ }^{5}$ Department of Industrial and Systems Engineering, Northern Illinois University, DeKalb, IL 60115, USA \\ ${ }^{6}$ School of Economics and Management, Shanghai Second Polytechnic University, Shanghai 201209, China
}

Correspondence should be addressed to Peng Liu; liup7802@163.com

Received 1 March 2015; Accepted 1 March 2015

Copyright (c) 2015 Peng Liu et al. This is an open access article distributed under the Creative Commons Attribution License, which permits unrestricted use, distribution, and reproduction in any medium, provided the original work is properly cited.

Scheduling is one of the active areas of discrete optimization that plays a crucial role in manufacturing and service industries and is probably its driving force today. The scheduling theory is developed largely by researchers in management science, industrial engineering, and operations research. There is still a lively interest in classical models incorporating new features that reflect modern industrial processes. The new scheduling problems arising from practical production processes have spawned a new area in scheduling field.

The main objective of this special issue is to present the original research articles on new theory development, innovative modelling and analysis, and novel applications in the field of scheduling. All authors were encouraged to focus on scheduling games, multiagent scheduling, multicriteria scheduling, supply chain scheduling, scheduling with batching, scheduling with variable processing times, coordinated scheduling with production and transportation, scheduling under resource constraints, and scheduling in flexible manufacturing and service systems.

The special issue received high-quality submissions. All of them were peer-reviewed according to high standards of this journal. The accepted papers represent excellent work that spans across a wide variety of cutting edge scheduling problems and applications.

In the paper entitled "Genetic Algorithm and Its Performance Analysis for Scheduling a Single Crane," X. Xie et al. study a single crane scheduling problem arising in the coldrolling material warehouse in an iron and steel enterprise. One overhead crane is used to perform all the transportation and shuffling operations. Since the problem is shown to be NP-hard, a genetic algorithm (GA) is proposed. They identify some analytical properties which enable them to develop an effective heuristic algorithm as initial solutions of the GA. They further analyze its performance from the worstcase point of view. To evaluate the average performances, a numerical test compared with some existed methods is carried out and its results show the good quality of the proposed algorithm.

In the paper entitled "Robust Proactive Project Scheduling Model for the Stochastic Discrete Time/Cost TradeOff Problem," H. Li et al. consider the project budget version of the stochastic discrete time/cost trade-off problem (SDTCTP-B) from the viewpoint of the robustness in the scheduling. By modeling the activity time and cost using interval numbers, they propose a proactive project scheduling model for the SDTCTP-B based on robust optimization theory. They convert their model into its robust counterpart using a form of the mixed-integer programming model. Extensive experiments are performed on a large number of randomly generated networks to validate their model. Simulation is used to analyze the impact of different project network parameters on the schedule stability. 
In the paper entitled "A Local Search Algorithm for the Flow Shop Scheduling Problem with Release Dates," T. Ren et al. discuss the flow shop scheduling problem to minimize the makespan with release dates. By resequencing the jobs, a modified heuristic algorithm is obtained for handling large-sized problems. Moreover, based on some properties, a local search scheme is provided to improve the heuristic to gain high-quality solution for moderatesized problems. A sequence-independent lower bound is presented to evaluate the performance of the algorithms. A series of simulation results demonstrate the effectiveness of the proposed algorithms.

In the paper entitled "A Study on the Enhanced Best Performance Algorithm for the Just-in-Time Scheduling Problem," S. Chetty and A. O. Adewumi propose a new local search metaheuristic algorithm to solve the just-in-time (JIT) scheduling problem. This algorithm is called the enhanced best performance algorithm (eBPA). The currently problem setting is the allocation of a large number of jobs required to be scheduled on multiple and identical machines which run in parallel. The due date of a job is characterized by a window frame of time rather than a specific point in time. The performance of the eBPA is compared against tabu search (TS) and simulated annealing (SA). The results show that eBPA performs competitively well with both TS and SA in terms of best and average fitness values.

In the paper entitled "Optimal Control Problem of Converter Steelmaking Production Process Based on Operation Optimization Method," J. Zhang provides a dynamic operation optimization method for operating a converter to solve an optimal control problem. The classification method is based on the use of clustering. A real-time multiobjective operation optimization model is built by taking the minimum deviation between the actual data and the reference data by the optimal control indicator as objective function. DE (differential evolution) with improved strategy is used to solve the proposed model for obtaining the set values of each operating variable, which is beneficial for further control. Simulation of actual production data shows the feasibility and efficiency of the proposed method. That proves the proposed method solves the optimal control problem of converter steelmaking process as well.

In the paper entitled "A Hybrid Algorithm for the Permutation Flowshop Scheduling Problem without Intermediate Buffers," X. Liu et al. deal with the permutation flow shop scheduling problem without intermediate buffers and present a hybrid algorithm based on the scatter search and the variable neighborhood search. A variable neighbourhood based on the notion of job-block is developed, and the neighbourhood size can adaptively change according to the construction of the job-block. Extensive experiments on benchmark problems are carried out, and the results show that the proposed hybrid algorithm is powerful and competitive with the other powerful algorithms in the literature.

In the paper entitled "A DE-Based Scatter Search for Global Optimization Problems," K. Li and H. Tian develop a hybrid scatter search algorithm for continuous global optimization problems by incorporating the differential evolution algorithm to act as the new solution generation method.
This hybrid algorithm is called a DE-based SS algorithm (SSDE). To make the proposed hybrid algorithm more robust for different kinds of optimization problems, an adaptive selection mechanism is developed for multiple mutation strategies. A population reinitialization method is also used to help the algorithm to get out from local optimums. The computational results based on benchmark test problems show that the proposed SSDE is competitive or superior to some state-of-the-art algorithms in the literature.

In the paper entitled "Modelling and Optimizing an Open-Pit Truck Scheduling Problem," Y. Chang et al. address a special truck scheduling problem in the open-pit mine with different transport revenue consideration. A mixed-integer programming model is formulated to define the problem clearly and a few valid inequalities are deduced to strengthen the model. Some properties and two upper bounds of the problem are proposed. Based on these inequalities, properties, and upper bounds, a heuristic solution approach with two improvement strategies is proposed to resolve the problem. The numerical experiment demonstrates that the proposed solution approach is effective and efficient.

In summary, the eight papers represent some of the latest and most promising research results on scheduling problems and applications. This special issue demonstrates the theoretical and practical importance of further studies on scheduling problems and applications.

\section{Acknowledgments}

We are grateful to all of the authors for their contributions and the reviewers for their effort providing helpful comments and suggestions. The work is also supported by the National Natural Science Foundation of China (Grant nos. 71001074, 71101097, and 11001117) and the Program for Liaoning Excellent Talents in University (Grant nos. WJQ2013003, LJQ2012017).

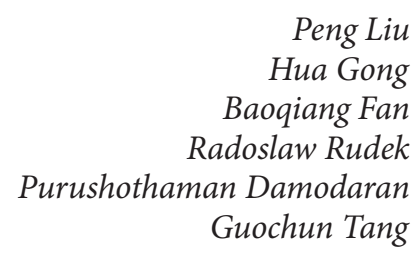

Peng Liu Hua Gong Baoqiang Fan Guochun Tang 


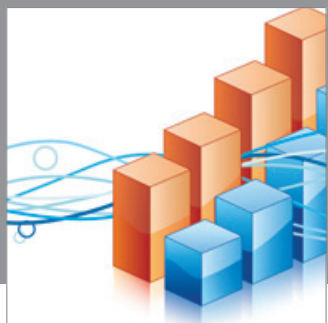

Advances in

Operations Research

mansans

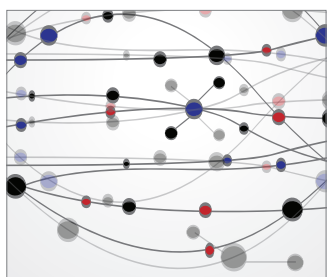

The Scientific World Journal
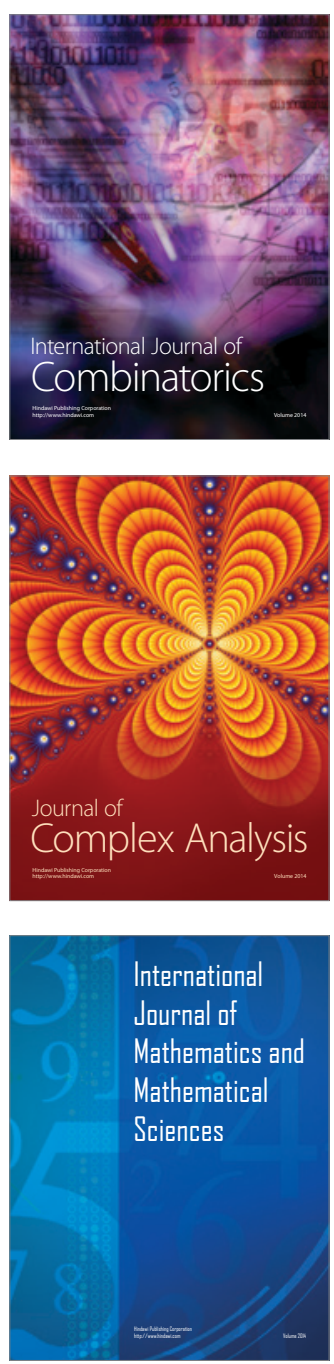
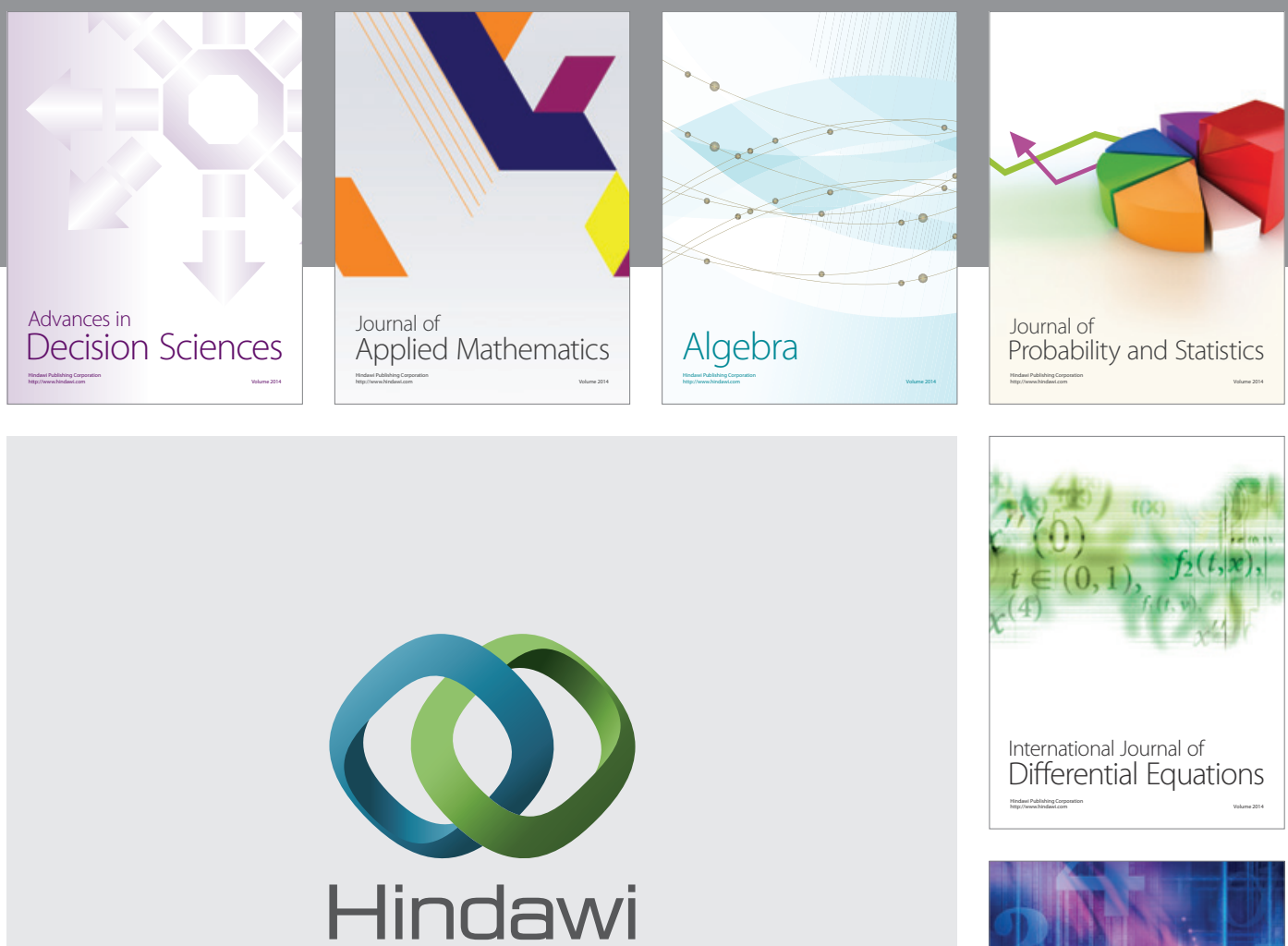

Submit your manuscripts at http://www.hindawi.com
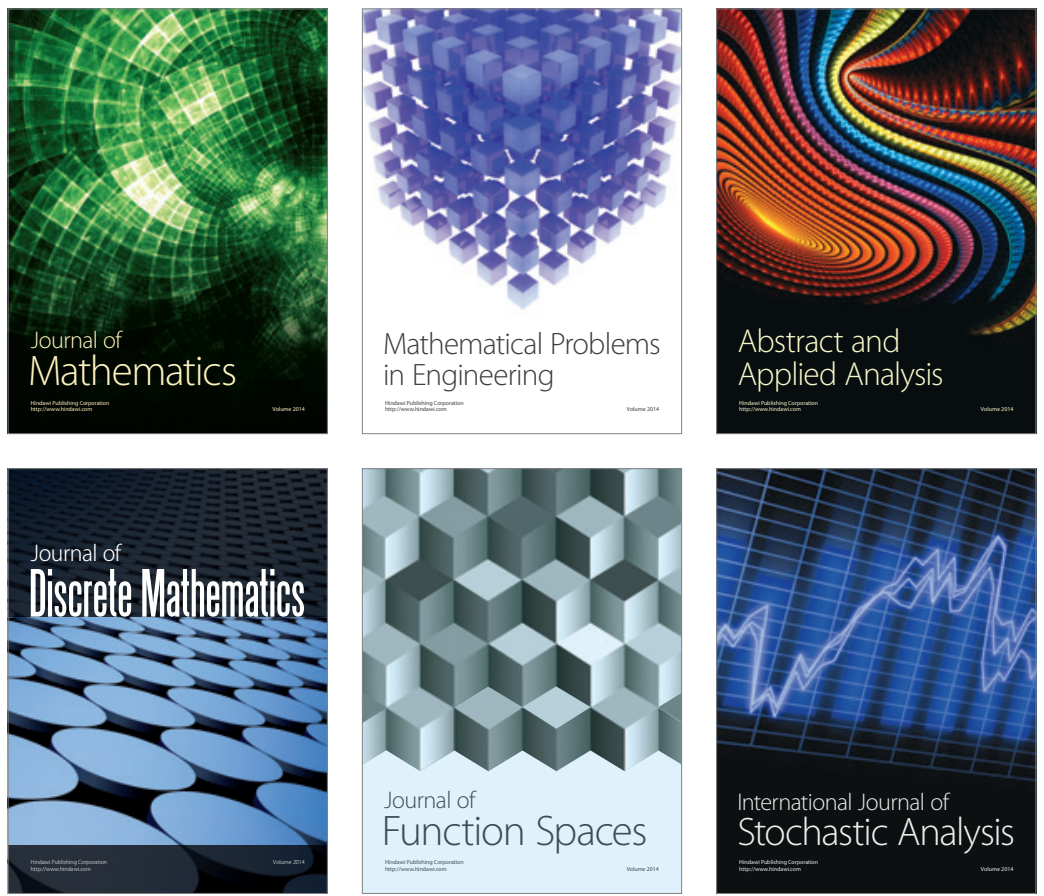

Journal of

Function Spaces

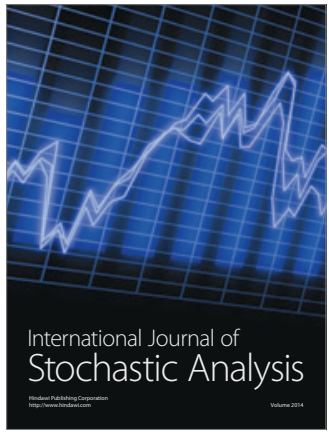

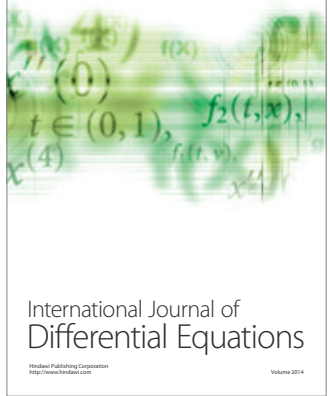
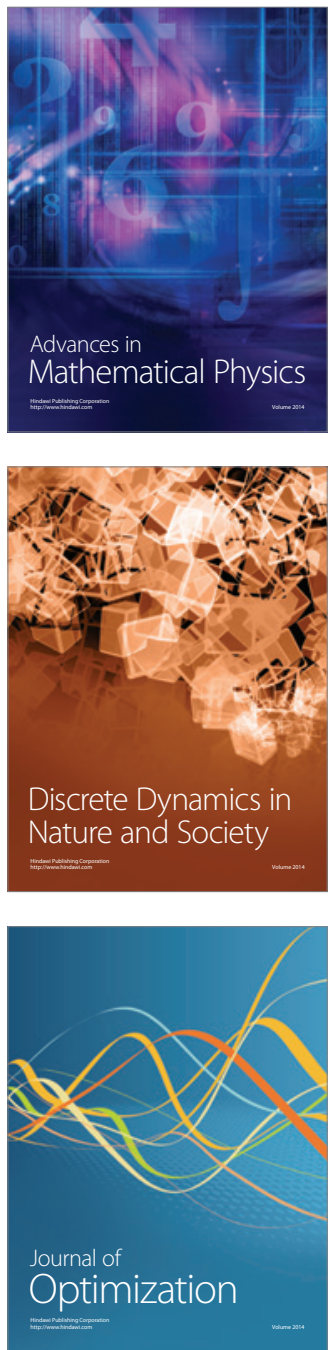